\title{
Cocaine-associated stroke: some new insights?
}

\author{
Sebastian Koch and Ralph L Sacco
}

Stroke can result from various toxic exposures including illicit drugs, with cocaine being one of the more frequently cited agents. Cocaine is known to be an important risk factor for ischemic and hemorrhagic stroke in young individuals. In young stroke populations, illicit drug use has been reported in 12-33\% of patients with stroke and is often implicated as the immediate cause of stroke; therefore, inquiring about and screening for illicit drug use is important, as such use is often underreported.

Cocaine abuse in the general population remains a widespread problem. In a US survey, $2.4 \%$ of the general population (age $>12$ ) reported cocaine use in the previous month (Substance Abuse and Mental Health Services Administration http://www.oas.samhsa.gov/ nhsda.htm). Given this high prevalence, it is surprising that more cocaine-associated cerebral infarcts are not reported. In the WashingtonBaltimore area, a region with reputed high levels of illicit drug use, 240 cocaine-associated strokes were diagnosed by 46 hospitals over a 4-year period (Sloan MA et al. [1998] Neurology 50: 1688-1693). Large inner city hospitals serving populations with high cocaine use admit about 5-10 individuals with cocaine-associated cerebral infarcts per year.

Numerous questions about the association between cocaine use and stroke remain unanswered. For example, what factors predispose certain individuals to develop cerebral infarction with cocaine? Why does stroke occur after a single initial use in some individuals, whereas others are spared despite repetitive chronic abuse? Also, if cerebral vascoconstriction occurs with a high incidence in healthy volunteers (Kaufman MJ et al. [1998] JAMA 279: 376-380), even after low-dose cocaine administration, why are neurovascular complications not more frequent?

Little is known about predispositions towards stroke in the setting of cocaine use. Pre-existing vascular risk factors such as hypertension and smoking might account for some individual

\section{Given the \\ manifold \\ mechanisms \\ of cocaine- \\ associated \\ infarction, it \\ comes as no \\ surprise to find \\ that no specific \\ treatment has \\ evolved}

S Koch is Associate

Professor of Neurology at the University of Miami, Miller School of Medicine, Miami, FL, USA, and $R L$ Sacco is an Advisory Board member of Nature Clinical Practice Neurology.

\section{Competing interests}

The authors declared no competing interests.

www.nature.com/clinicalpractice doi:10.1038/ncpneuro0925 susceptibility to ischemic stroke. In hemorrhagic stroke, underlying vascular pathology might be a contributory factor, although a role for preexisting vascular pathology as a risk factor for stroke related to drug use has not been uniformly reported (Sloan MA et al. [1998]).

Two Case Studies in this issue remind us of the complex relationship between cocaine and stroke and illustrate the variety of stroke mechanisms associated with cocaine use. MacEwen et al. highlight sympathomimetic effects, such as vasospasm, platelet activation and superimposed thrombosis. Less well known are the paracrine and immune effects of cocaine that can lead to a form of vasculopathy, as reported by Han et al.. In the patient described by Han et al., a definitive diagnosis of vasculitis remains elusive in the absence of histological confirmation. The blood oxygen level-dependent MRI vasomotor reactivity findings are nonspecific and could be a manifestation of the hemodynamic effects of large-vessel involvement.

Han et al.'s demonstration of vessel wall enhancement in the setting of cocaine use might be of considerable clinical interest. Extracranial vessel wall enhancement on high-resolution MRI has recently been found to have excellent sensitivity (81\%) and specificity (97\%) for the diagnosis of giant cell arteritis (Bley TA et al. [2007] AJNR Am J Neuroradiol 28: 1722-1727). There are also preliminary reports of intracranial vessel wall thickening and enhancement in cerebral vasculitic disorders.

Given the manifold mechanisms of cocaineassociated infarction, it comes as no surprise to find that no specific treatment has evolved. In the acute setting, we agree with MacEwen that thrombolytic thrombolytic therapy in select cases of thrombosis and embolism should be endorsed until further evidence emerges. For longer term secondary stroke prevention, antiplatelet agents, control of vascular risk factors, and, most importantly, abstinence from cocaine should be the focus of our treatment recommendations. 